Kong. Res. J. 2(2) : 13-15, 2015

ISSN 2349-2694

Kongunadu Arts and Science College, Coimbatore.

\title{
ZnO NEEDLE-LIKE STRUCTURES: SYNTHESIS AND CHARACTERIZATION
}

\author{
Sugapriya, $\mathbf{S}^{1^{*}}$, S. Lakshmi ${ }^{1}$, C. K. Senthil kumaran ${ }^{2}$, B. Chandarshekar ${ }^{2}$ and R. Ranjithkumar ${ }^{3}$ \\ ${ }^{1}$ Department of Chemistry, Coimbatore Institute of Technology, Coimbatore, Tamil Nadu, India. \\ 2Department of Physics, Nanotechnology Research Lab, Kongunadu College of Arts and Science, Coimbatore, \\ Tamil Nadu, India. \\ ${ }^{3}$ Bionano Research Centre, The Nilgiris Education and Research Foundation, The Nilgiris, Tamil Nadu, India. \\ *E.mail: sugapriya0314@gmail.com
}

\section{ABSTRACT}

We report in this paper, the structural and I-V properties of $\mathrm{ZnO}$ nano needle-like structure synthesized by Co-precipitation method. X-ray diffraction (XRD) result shows that the $\mathrm{ZnO}$ nano needle-like structure with hexagonal phase and no secondary phase was observed. The crystallite size has been calculated by Scherrer's equation which was found to be in the range 40-60 nm. SEM images reveal that $\mathrm{ZnO}$ nano needle-like structure has the length of $\sim 5.5 \mu \mathrm{m}$ and base of $\sim 5 \mu \mathrm{m}$ are consistent with the results from SEM investigations. I-V characteristics have been carried out to study the conducting behaviour of the prepared ZnO nano needle-like structures.

Keywords: ZnO nano needle-like structure, Co-precipitation, I-V characteristics, SEM.

\section{INTRODUCTION}

Nanoscale semiconductor materials have attracted great interests of researchers because of their importance not only in fundamental research areas but also in practical applications. $\mathrm{ZnO}$ nanostructures have been studied intensively and extensively over the last decSade not only for their remarkable chemical and physical properties, but also for their current and future diverse technological applications. $\mathrm{ZnO}$ is a typical inorganic semiconductor and piezoelectric material; this material has a direct wide band gap of $3.37 \mathrm{eV}$ and a large exciton binding energy of $60 \mathrm{meV}$ at room temperature (Ozgur et al., 2005). It has enormous applications in electronic and electromechanical devices (Wang, 2009), such as ultraviolet (UV) lasers (Kota et al., 2011), high performance nanosensors (Zhou et al., 2009), solar cells (Weintraub et al., 2009), piezoelectric nano generators (Yang et al., 2009), and nanopiezotronics (Wang, 2008). In order to grow one-dimensional (1D) $\mathrm{ZnO}$ nanostructures, various techniques have been developed like wet chemical methods (Narkiewicza et al., 2008), physical vapour deposition (Onur et al., 2011), pulsed laser deposition (Hong et al., 2009), sputtering (Dang, 2007). This article gives a comprehensive overview of the progress that has been made within the context of one-dimensional (1D) $\mathrm{ZnO}$ nanostructures synthesized via chemical precipitation method.

\section{EXPERIMENTAL}

$\mathrm{ZnO}$ nano needle have been prepared using the required precursors by chemical precipitation method. An aqueous solution of $1 \mathrm{M}$ Zinc acetate dihydrate $\left\{\mathrm{Zn}\left(\mathrm{CH}_{3}(\mathrm{COO})\right)_{2} .2 \mathrm{H}_{2} \mathrm{O}\right\}$ dissolved in water and stirred for about $30 \mathrm{~min}$ at room temperature. Sodium hydroxide $(\mathrm{NaOH})(0.7 \mathrm{M})$ was added drop wise to the above mentioned solution. The colour of the solution changed into milk white coloured, indicating the formation of $\mathrm{ZnO}$ nano particles in the solution. The solution was stirred for $10 \mathrm{~h}$ at room temperature. After $10 \mathrm{~h}$ the supernatants were removed and the deposited precipitate was centrifuged and washed with water and ethanol several times. The samples were then suspended in ethanol and allowed to age for $6 \mathrm{~h}$ without stirring. After centrifugation, the samples were then dried in oven at $60^{\circ} \mathrm{C}$ for $2 \mathrm{~h}$. Then, the as prepared $\mathrm{ZnO}$ nanoparticle is placed in the middle of a muffle furnace in silica crucible. The samples have been annealed at $400^{\circ} \mathrm{C}$ for one hour.

\subsection{Characterization of $\mathrm{ZnO}$ nanoparticles}

X-ray diffraction studies have been carried out using PANalytical x-ray diffractometer and surface morphology and the compositional analysis of the samples has been studied using scanning electron microscope (JEOL JSMS 800-V) and energy dispersive analysis studied using the prepared $\mathrm{ZnO}$ nano needle-like structured samples have been recorded using a JEOL JEM2100 microscope. I-V characteristics of $\mathrm{ZnO}$ nano needle-like structured 
samples have been recorded by using four-probe method.

\section{RESULTS AND DISCUSSION}

Fig. 1 shows the X-ray diffraction patterns of the $\mathrm{ZnO}$ nano needle-like structure. The diffraction peaks at $2 \theta$ (degrees) of $31.63^{\circ}, 34.61^{\circ}, 36.32^{\circ}$, $47.66^{\circ}, 56.94^{\circ}, 62.97^{\circ}, 66.57^{\circ}, 68.12^{\circ}, 69.48^{\circ}, 72.11^{\circ}$ and $72.26^{\circ}$ are respectively indexed as the (100), (002), (101), (102), (110), (103), (200), (112), (201), (004) and (202) planes of $\mathrm{ZnO}$. All the diffraction peaks in the $2 \theta$ range measured corresponds to the hexagonal structure of $\mathrm{ZnO}$ with lattice constants $\mathrm{a}=$ $3.253 \AA$ and $c=5.214 \AA$ and are in good agreement with those on the standard data card (JCPDS card No. 36-1451).

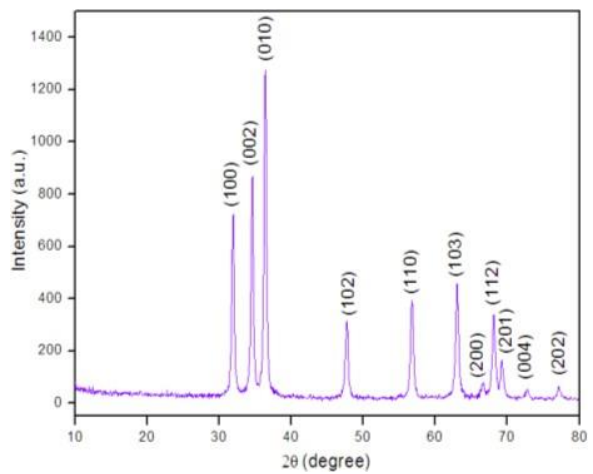

Fig. 1. X-ray diffraction pattern of as prepared ZnO nano needle-like structure

The sharpness of the diffraction peaks suggests that the product is well crystallized. The crystallite size of $\mathrm{ZnO}$ is calculated using Scherrer's equation

$$
D=\frac{K \lambda}{\beta \operatorname{Cos} \theta}
$$

where, $\mathrm{D}$ is the crystallite size, $\mathrm{K}$ is a constant taken to be $0.94, \lambda$ is the wavelength of the $\mathrm{X}$-ray radiation, $\beta$ is the full width at half maximum and $\theta$ is the angle of diffraction. The crystallite size has been calculated and is found to be in the range 40-60 nm for as prepared $\mathrm{ZnO}$ nano needle-like structure.

Fig. 2a displays an SEM image of the sample prepared with zinc acetate and sodium hydroxide as reactants under conventional conditions, from which it can be seen that there are many nanorods with flat ends, are seen to arise from centre, it gives the appearance of a needle, their average diameter are 20 to 30 nanometers and length varies from 70 nanometers to $5 \mu \mathrm{m}$ and the average size of whole needle is $5 \mu \mathrm{m}$ shows needle-like structures. The size of the complex structure and the diameter and length of the $\mathrm{ZnO}$ nano needle-like structure are consistent with the results from SEM investigations. Energy dispersive X-ray analysis (EDS) of ZnO nano needle-like structures are shown in Fig. 2b. The chemical constituents present in the $\mathrm{ZnO}$ sample are of $\mathrm{Zn}-49.65 \%$ and $0-50.35 \%$. In the EDS, $\mathrm{Zn}$ and 0 are the element detected, indicating that the sample is highly pure.

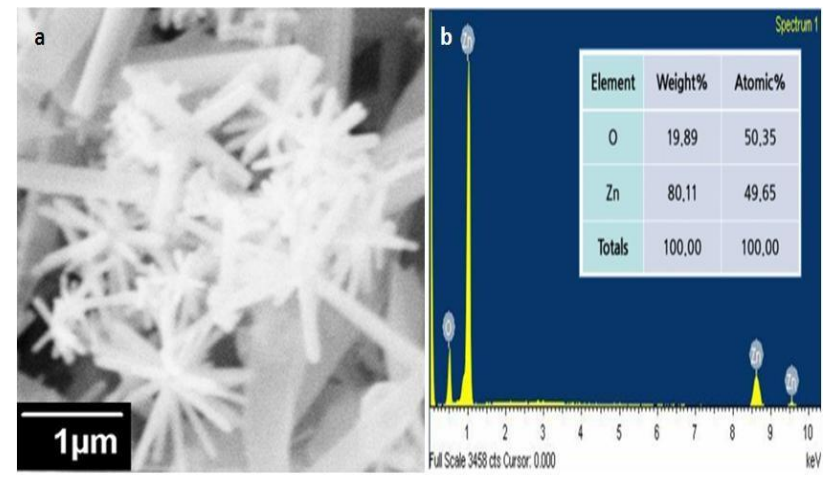

Fig. 2. a)SEM images of ZnO nanoparticles and b) EDS spectra of ZnO nanoparticles

For I-V measurements in bulk, pellets of 13 $\mathrm{mm}$ diameter and thickness $\approx 1 \mathrm{~mm}$ were prepared under a load of 5 tons. These pellets were used in Four-Probe method. The I-V characteristics of the samples were studied at the room temperature as well as at various temperatures $(125 \mathrm{~K}, 200 \mathrm{~K}, 273 \mathrm{~K}$, $300 \mathrm{~K}, 350 \mathrm{~K}$ and $400 \mathrm{~K}$ ) using the Four-Probe method. The temperature dependence of resistivity was measured at constant current by varying the temperature continuously. The silver paste was used for Ohmic contact between the sample and the copper probes. DC voltage across the electrodes was measured by varying the current. I-V plots are shown in Fig. 3(a) which shows the temperature dependence of I-V characteristic of $\mathrm{ZnO}$ nano needlelike structure as a representative case.
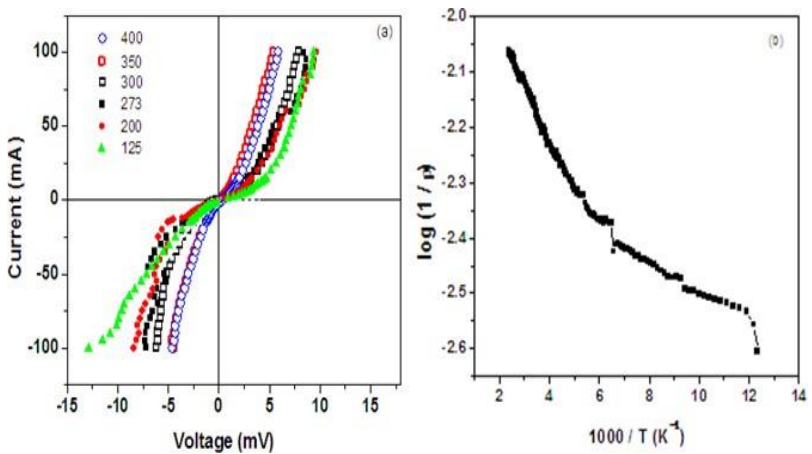

Fig. 4 (a) I-V characteristics (b) Conductivity Vs Temperature ZnO nano needle-like structure sample 
I-V characteristics of the samples are measured in presence of argon gas at low as well as at high temperatures. Liquid Nitrogen is used for lowering the temperature. Argon gas is necessary to eliminate the moisture content otherwise the moisture present in air will change the electrical properties of the sample (especially at low temperatures). Fig. 3(a) depicts the characteristics of pure $\mathrm{ZnO}$ at the constant current, where the voltage decreases as we go on lowering the temperature. The conductivity of the sample increases with increasing temperature, as shown in the Fig. 3(b) and in the Table 1.

Table 1. Temperature with Conductivity

\begin{tabular}{lll}
\hline $\mathbf{T}(\mathbf{K})$ & $\sigma\left(\boldsymbol{\Omega}^{-1}\right)$ & $\mathbf{R}(\boldsymbol{\Omega})$ \\
\hline 400 & 0.66511 & 1.5035 \\
350 & 0.77082 & 1.2973 \\
300 & 0.85095 & 1.1751 \\
273 & 1.08472 & 0.9219 \\
200 & 1.25094 & 0.7994 \\
125 & 1.66917 & 0.5991 \\
\hline
\end{tabular}

\section{CONCLUSION}

The structural and electrical properties of $\mathrm{ZnO}$ nano needle-like structure was synthesized by a simple chemical bath deposition method. The sizes of the needle-like structures are about $\sim 5.5 \mu \mathrm{m}$ on an average. The length and breadths of the needle-like structure are about $\sim 5.5 \mu \mathrm{m}$ and $\sim 5 \mu \mathrm{m}$. The size of the complex structure and the length and breadths of the $\mathrm{ZnO}$ nano needle-like structure are consistent with the results from SEM investigations. The conductance of the $\mathrm{ZnO}$ nano needle-like structure was estimated from the I-V characteristic from lower temperature to higher temperature. The conductance of the sample increases with decreasing temperature. The increase in the conductivity can be attributed to the increase in the electrical property.

\section{REFERENCES}

Dang, W.L., Y.Q. Fu, J.K. Luo, A.J. Flewitt and W.I. Milne, (2007). Deposition and characterization of sputtered ZnO films. SuperlatticesMicrostruct. 42: 89-93.
Hong, J.I., J. Bae, Z.L. Wang and R.L. Snyder, (2009). Room temperature, texture-controlled growth of $\mathrm{ZnO}$ thin films and their application for growing aligned $\mathrm{ZnO}$ nanowire arrays. Nanotechnol. 20: 085609.

Kota, O., Kazuki Kubo, Tetsuya Shimogaki, Daisuke Nakamura, Mitsuhiro Higashihata and Tatsuo Okada, (2011). Lasing characteristics of $\mathrm{ZnO}$ nanosheet excited by ultraviolet laser beam, Adv. Mat. Lett., 2(5): 2011, 354-357.

Narkiewicza, U., D. Siberaa, I. Kuryliszyn-Kudelskab, L. Kilanski, W. Dobrowolskib and N. Romcevi, (2008). Synthesis by Wet Chemical Method and Characterization of Nanocrystalline ZnO Doped with $\mathrm{Fe}_{2} \mathrm{O}_{3}$. Acta Physica Polonica A 113: 16951700 .

Onur, T. and J. Juhala, (2011). ZnO Nanowire Growth by Physical Vapor Deposition, IEEE International Conference on Nanotechnology Portland Marriott August 15-18, Portland, Oregon, USA.

Ozgur, U., Y.L., Alivov, C. Liu and A. Teke, (2005). Reshchikov, M.A.; Dogan, S.; Avrutin, V.; Cho, S. J.; Morkoc, H. A comprehensive review of $\mathrm{ZnO}$ materials and devices. J. Appl. Phys. 98: 041301.

Wang, Z.L. (2008). Towards self-powered nanosystems: Fromn nanogenerators to nanopiezotronics. Adv. Funct. Mater. 18: 35533567.

Wang, Z.L. (2009). ZnO nanowire and nanobelt platform for nanotechnology. Mat. Sci. Eng. R 64: 33-71.

Weintraub, B., Y.G. Wei and Z.L. Wang, (2009). Optical fiber/ nanowire hybrid structures for efficient three-dimensional dye-sensitized solar cells. Angew. Chem. Int. Ed. 48: 8981-8985.

Yang, R.S., Y. Qin, L. Dai, and Z.L. Wang, (2009). Power generation with laterally packaged piezoelectric fine wires. Nat. Nanotechnol. 4: 34-39.

Zhou, J., Y. Gu, Y.F. Hu, W.J. Mai, P.H. Yeh, G. Bao, A.K. Polla and D.L. Wang, (2009). Gigantic enhancement in response and reset time of $\mathrm{ZnO}$ UV nanosensor by utilizing Schottky contact and surface functionalization. Appl. Phys. Lett. 94: 191103. 